Relations industrielles

Industrial Relations

\title{
Essentials of Management, par Joseph L. Massie, Prentice-Hall, Inc. Englewood Cliffs, N.J., 1964, 180 pages.
}

\section{Bertrand Belzile}

Volume 19, numéro 3, juillet 1964

URI : https://id.erudit.org/iderudit/1021284ar

DOI : https://doi.org/10.7202/1021284ar

Aller au sommaire du numéro

Éditeur(s)

Département des relations industrielles de l’Université Laval

ISSN

0034-379X (imprimé)

1703-8138 (numérique)

Découvrir la revue

Citer ce compte rendu

Belzile, B. (1964). Compte rendu de [Essentials of Management, par Joseph L. Massie, Prentice-Hall, Inc. Englewood Cliffs, N.J., 1964, 180 pages.] Relations industrielles / Industrial Relations, 19(3), 406-407.

https://doi.org/10.7202/1021284ar

Tous droits réservés (C Département des relations industrielles de l’Université Laval, 1964
Ce document est protégé par la loi sur le droit d'auteur. L’utilisation des services d'Érudit (y compris la reproduction) est assujettie à sa politique d'utilisation que vous pouvez consulter en ligne.

https://apropos.erudit.org/fr/usagers/politique-dutilisation/ 
Relations Industrielles, vol. 19 , No 3

II arrive que l'individu expérimente des échecs répétés dans la satisfaction de ses besoins. C'est là l'origine de la frustration, de l'anxiété et du conflit au sein des individus et des groupes. La nature de ces revers psychologiques et leur influence sur le com. portement des individus au travail forment la troisième partie. Par exemple, un certain état d'anxiété pousse un individu à amé. iiorer son rendement, alors qu'un degré élevé d'anxiété peut l'empêcher d'occomplir sa tôche. Les exigences imposées por la direction peut soulever divers étots d'anxiété qui influencent le comportement des travaiileurs.

Une entreprise qui veut prospérer n'est pas sans opérer à un moment ou l'autre des changements nécessaires. Ceci entraîne chez les individus la nécessité d'adopter des attitudes et des formes de conduite nouvelles. L'introduction des changements occasionne aussi une résistance dont la nature diffère avec les niveoux organisotionnels Les quatrième et cinquième parties traitent des conditions qui donnent naissance aux changements, des principes et focteurs essentiels qui facilitent l'acquisition de nouvelles attitudes et conduites. Les auteurs fournissent aux dirigeants quelques guides pour l'application des principes et la création des conditions susceptibles de faciliter l'adaptotion des individus. L'efficacité des shèmes de participation et de formation des cadres en vue de faciliter l'odoption est étudiée et critiquée.

La cernière partie de l'ouvrage fait une distinction entre le processus de solution d'un problème et la théorie de la décision. C'est une description des phases du processus, et des facteurs qui en conditionnent l'efficacité. Les auteurs font des suggestions très concrètes pour l'établissement $d$ 'un programme d'entraînement en vue d'améliorer le processus. Les orticles de Simon, Cyert, Trow et d'Edwards sur la théorie de la décision méritent d'être signalés, puisqu'ils démontrent d'une façon empirique les limites des modèles économiques de prise de décision ou sein de la grande entreprise. Edwards présente un modèle de maximisation de l'utilité subjective onticipée. Comme la prise de décision fait appel à l'imagination et à la pensée créatrice, il est bon de connaître les critères qui permettent de détecter le talent créoteur de certains individus. Beaucoup de décisions sont prises aussi par des groupes. Les résultats de recherches tendent à démontrer ia supériorité du groupe sur l'individu dans la prise de décision. On retrouve ici un inventaire des variables importantes qui conditionnent l'efficacité du «group problem solving \. La qualité du leadership, le réseau de communication, la cohésion du groupe, la personnalité et le status des individus qui le compose sont autant de variables dont il faut tenir compte lorsqu'on s'interroge sur l'efficacité de la prise de décision par un groupe.

Un administrateur qui ne possède pas quelques notions de psychologie ou de sociologie trouvera ce volume difficile d'accès. Mais ceux qui s'intéressent à la dynamique de groupe, à la formation des cadres ou à l'administration du personnel en général peuvent en faire une lecture profitable.

Les définitions de concepts, les modèles théoriques, les résultats de recherches l'emportent sur les applications pratiques qui tiennent très peu de place dans le volume. Les auteurs avaient probablement l'intention de laisser à l'administroteur le soin de faire les applications concrètes qu'il jugera utiles. Comme les «sciences du comportement * sont jeunes et qu'on ne connaît pas encore toute leur richesse d'application concrète, cette lacune mentionnée plus haut devient plus facilement explicable.

\section{Laurent Bélanger}

Essentials of Management, par Joseph $L$. Massie, Prentice-Hall, Inc. Englewood Cliffs, N.J., 1964, 180 pages.

Ce petit volume fait le point sur les connoissances présentes en administration. II constitue une sorte de vademecum dans ce domaine. De plus, son mérite ne réside pos uniquement dans la description des connaissances, mais plutôt dans leur présentation critique et systématique. En effet, l'auteur expose une conception moderne de l'administration qui accorde une importance privilégiée au processus de la prise de décision et qui foit lorgement oppel à plusieurs disciplines diverses. Quant à la forme, le texte est vivant et le style clair et concis; on trouve également une bibliogrophie très sélective à la fin de chaque chapitre.

Dans une première partie, l'auteur décrit I'arrière-plan de l'administration: so nature et son importance, le développement de la pensée odministrotive et les dimensions morales et sociales de l'administration.

Lo deuxième portie couvre les fonctions du processus odministratif. L'outeur en distingue sept: prise de décision, organisation et 《staffing 》, planification et contrôle; communication et direction. D'après lui, la plupart des devoirs des administrateurs impliquent prises de décision d'une sorte ou 
d'une outre, et ainsi on pourroit discuter des six autres fonctions sous le titre général de prise de décision.

Lo partie suivante expose les éléments des disciplines fondomentales pour les administrateurs: économie de gestion, comptabilité de gestion, relations humaines et sciences du comportement et enfin méthodes quantitatives d'analyse

Dans une dernière portie, on trouve des applications des concepts administratifs et des techniques d'onalyse oux activités d'une entreprise. L'outeur distingue à cet effet les trois octivités fonctionnelles classiques, d̀ savoir: administration manufacturière, administration financière et administration en \& marketing $>$.

\& Essentials of Manogement est un volume qu'il faut lire et conserver à la portée de la main

\section{Bertrond Belzile}

\section{How to Negociate the Labor Agreement - An Outline Summary of Tested Bargaining Practice, par Bruce Morse, Trends Publish-} ing, Co., Detroit 1963. 62 pages.

Cette petite ploquette de soixante-deux pages n'est pas autre chose qu'un compendium sur la technique de la négociation collective. C'est en quelque sorte un guide simplifié de la pratique de la négociation, de ses recettes, utilisées par les directions d'entreprises oyant à négocier ovec les syndicats.

L'auteur ne discute rien au niveau des grandes politiques du <management $>$ et ne fait aucune place à la «philosophie $\gg$. L'idée de base de ce petit ouvrage est simplement de mettre entre les mains du négociateur patronal un sommaire des principoux faits et méthodes de nature ò lui indiquer, d'une façon détaillée et ordonnée, les principaux jalons à poser à ses démarches lorsqu'il se trouve d̀ faire face oux représentants du syndicat.

II lui indique où trouver les faits, ies données nécessaires à son action, comment les organiser et les utiliser de la foçon la plus efficace possible.

Ce n'est même pas un traité sur la convention collective, comme nous en connaissons plusieurs, où souvent l'étude des différentes composantes de la négociation et de la convention sont étudiées méthodiquement, et où on ajoute des séries de problèmes, de «cas $»$ devant être discutés par les étudionts.
Ici, rien de celà, mais en revanche des informations détaillées quant ò l'importance des principales clauses, à la cueillette des données, à leur calcul, à l'organisation et à la présentation des contre-propositions patronales, aux attitudes à développer ou à bannir chez le négociateur, aux aspects légaux et stratégiques à respecter.

Somme toute un vade mecum très utile à la fois pour le négocioteur professionnel à qui il peut servir d'élément de comparaison ovec les pratiques de sa propre organisation, et au responsable d'une entreprise dépourvue de personnel spécialisé aux prises ovec une négociation et manquant d'expérience et de conseil. A ce dernier surtout, ce petit précis peut se révéler d'une très grande utilité.

\section{Jean-Réal Cardin}

\section{Quelques aspects du Droit de la Province de} Québec, par Louis Baudoin et al. Ouvrage publié ovec le concours du CN.R.S., Publication du Centre français de Droit comparé, Editions Cujos, Paris, 1963, $279 \mathrm{pp}$

L'ouvrage est un recueil d'études portant sur différents aspects du droit tel qu'il existe de nos jours dans le Québec. Publié avec le concours du Centre national de la Recherche scientifique et préfacé par l'éminent juriste français Léon Julliot de la Morandière, ce recueil est destiné avant tout ò mettre le monde juridique français au courant des principoles lignes de force et des problèmes les plus caractéristiques du droit québécois contemporain

Il est l'oeurre de juristes et de praticiens conadiens du droit, à la tête desquels il faut mentionner $M$. le professeur Louis Boudoin, de la Faculté de Droit de I'Université McGill

Comme le professeur Baudoin le souligne dans son étude, la Province de Québec est un laborotoire merveilleux de droit comporé, étont située géographiquement et historiquement, aussi bien dans l'ordre culturel que juridique, aux confins de civilisations différentes mais en quelque sorte complémentaires, si on sait les envisoger dans l'optique d'un monde en constante progression vers une plus grande solidarité.

Notre héritoge fronçais, ovec so mentolité, ses institutions fondamentales comme la famille et la paroisse, son droit civil codifié en 1866 et so langue; l'apport non équivoque des institutions politiques, constitutionnelles et de droit public de l'élément 